\title{
Indicadores de saúde para idosos: comparação entre o Brasil e os Estados Unidos
}

\author{
Mônica Rebouças ${ }^{1}$ e Maurício Gomes Pereira²
}

Como citar Rebouças M, Pereira MG. Indicadores de saúde para idosos: comparação entre o Brasil e os Estados Unidos. Rev Panam Salud Publica. 2008;23(4):237-46.

RESUMO Objetivo. Identificar no Brasil indicadores equivalentes aos utilizados nos Estados Unidos para avaliar a população com idade de 65 anos ou mais no ano de 2000 e comparar a situação nos dois países.

Método. Este é um estudo descritivo e transversal, baseado em dados secundários. A partir de uma lista de indicadores de bem-estar empregada nos Estados Unidos, equivalentes brasileiros foram pesquisados nos sites de instituições públicas de saúde ou de planejamento. Também foram utilizados dados de pesquisas nacionais e, se necessário, foram feitas reanálises de bases de dados.

Resultados. Dos 31 indicadores que compõem a lista norte-americana, somente para três não foi encontrado um equivalente brasileiro: comprometimento de memória, atividade social e crimes violentos. Nos Estados Unidos, o número de idosos foi maior e a escolaridade desse grupo foi mais alta. Os dois países foram semelhantes em termos de condição de saúde e expectativa de vida aos 65 anos. Maiores taxas de óbito por doença cerebrovascular e diabetes foram registradas no Brasil e por neoplasias malignas nos Estados Unidos. A morbidade auto-referida por artrite e por depressão foi mais freqüente nos Estados Unidos, assim como as incapacidades. A autopercepção de bom estado de saúde foi superior no Brasil, ao passo que os indicadores de hábitos de vida e os fatores de risco revelaram uma melhor situação nos Estados Unidos.

Conclusões. Se o poder discriminatório dos indicadores utilizados for de fato adequado, as condições de saúde dos idosos no ano de 2000 eram próximas nos dois países.

Palavras-chave Indicadores, idoso, saúde do idoso, Brasil, Estados Unidos.

Como outros países, o Brasil passa, atualmente, por um considerável envelhecimento populacional (1-3). São reflexos desse envelhecimento as inicia-

1 Universidade Federal do Ceará, Disciplina de Geriatria, Fortaleza, Ceará, Brasil. E-mail: monica reboucas@terra.com.br

2 Universidade Católica de Brasília, Pós-Graduação de Gerontologia, e Universidade de Brasília. Brasília, DF, Brasil. E-mail: mauriciogpereira@gmail.com tivas relacionadas ao estabelecimento de indicadores para avaliar a condição de saúde dos brasileiros idosos. Um exemplo é o Comitê Temático Interdisciplinar da Rede Interagencial de Informações para a Saúde (RIPSA) (4). Essa rede, que atua com base em um acordo de cooperação entre o Ministério da Saúde do Brasil e a Organização Pan-Americana da Saúde (OPAS), en- globa representantes de numerosas entidades e disponibiliza um conjunto de indicadores selecionados nas áreas demográfica, socioeconômica, de mortalidade, de morbidade e de fatores de risco, de recursos e de cobertura (5). Nos Estados Unidos, por sua vez, um fórum de agências governamentais publicou e atualiza periodicamente um relatório de fácil manuseio, não 
técnico, que engloba diversos relatos técnicos produzidos pelas agências vinculadas à iniciativa e que abrange diversos aspectos de saúde e bemestar dos idosos (6).

$\mathrm{O}$ envelhecimento acarreta mudanças no padrão das doenças e na freqüência das incapacidades (7). Esse fenômeno justifica a troca de experiências sobre as estratégias para a promoção do bem-estar de idosos em diferentes países, mesmo que provindas de contextos diversos $(8,9)$. Assim sendo, o objetivo do presente estudo foi identificar no Brasil um grupo de indicadores equivalentes aos utilizados nos Estados Unidos para avaliar a população idosa e comparar os dois países quanto a essas informações.

\section{MATERIAIS E MÉTODOS}

A pesquisa teve delineamento descritivo e transversal, sendo baseada em dados secundários. As informações referem-se a pessoas com 65 anos ou mais nos Estados Unidos (10). No Brasil, esse grupo etário foi utilizado sempre que possível. Porém, como os brasileiros são considerados idosos a partir dos 60 anos (11), para alguns indicadores só foi possível obter informações sobre esse grupo mais amplo.

Um fórum norte-americano sobre estatísticas relacionadas ao envelhecimento (6) utilizou, para refletir a situação da população idosa no ano de 2000, um total de 31 indicadores. A escolha desses indicadores seguiu os seguintes critérios:

- facilidade de compreensão por uma grande parcela de público;

- origem dos dados (sistemas de informação nacionais, de responsabilidade do governo);

- objetividade (dados baseados em pesquisa);

- equilíbrio entre os indicadores (nenhuma área é mais privilegiada do que outra);

- periodicidade da coleta de dados, o que permite o estabelecimento da tendência e da evolução da característica que está sendo monitorada pelo indicador; e
- representação de grandes segmentos da população idosa (ao invés de se referirem a um grupo em particular).

O presente estudo investigou indicadores de saúde brasileiros semelhantes aos utilizados pelo fórum norte-americano, referentes ao ano de 2000 (6). No Brasil, as informações acerca dos 31 indicadores foram buscadas inicialmente no site da RIPSA (12). As informações foram complementadas por meio de pesquisa em outras fontes ou diretamente nos microdados do Instituto Brasileiro de Geografia e Estatística (IBGE) e do Ministério da Saúde.

Os inquéritos populacionais brasileiros de cobertura nacional que serviram de referência foram a Pesquisa Nacional por Amostra de Domicílios (PNAD), realizada em 1998 e 2003; a Pesquisa de Orçamentos Familiares (POF), em 1995 e 1996 e em 2002 e 2003; e o Inquérito Domiciliar sobre Comportamentos de Risco e Morbidade Referida de Doenças e Agravos Não-Transmissíveis, de 2002 e 2003 (13-22). Os indicadores não localizados nas etapas anteriores foram buscados em publicações especiais e em periódicos científicos (23-29). Os 31 indicadores foram subdivididos em demográficos, socioeconômicos, condições de saúde, hábitos de vida e fatores de risco e assistência à saúde.

$\mathrm{O}$ relatório do Federal Interagency Forum on Aging-Related Statistics (6) sobre os indicadores dos idosos nos Estados Unidos, usado aqui como referência, contém séries históricas. No presente estudo, os dados estão restritos ao ano de 2000 ou ao ano mais próximo para ambos os países. Os nomes em inglês dos indicadores dos Estados Unidos foram mantidos. Na apresentação dos dados brasileiros, utilizou-se a designação em português adotada nas fontes pesquisadas. A falta de correspondência entre as categorias usadas em cada país foi assinalada nas tabelas.

\section{RESULTADOS}

Somente para três dos 31 indicadores utilizados pelos Estados Unidos no ano de 2000 não foi encontrada uma equivalência brasileira. São eles: o comprometimento de memória, a atividade social e os crimes violentos.

A população de norte-americanos com 65 anos ou mais, em números absolutos, era de 35 milhões; no Brasil, essa população era de 10 milhões de indivíduos, ou seja, 3,5 vezes menor (tabela 1). Em termos relativos, a população idosa dos Estados Unidos representava $13 \%$ da população daquele país, contra $6 \%$ de brasileiros com 65 anos ou mais de idade.

Quanto à distribuição racial, os números indicaram que havia uma predominância de idosos de cor branca em ambos os países. Os idosos, em sua maioria, eram casados, embora, no Brasil, mais idosos tenham respondido ao censo que eram solteiros. Em termos de escolaridade, apenas $10 \%$ dos idosos brasileiros concluíram o ensino médio ou superior, proporção muito menor do que nos Estados Unidos, onde a taxa alcançou $67 \%$. Com relação aos arranjos familiares, os idosos residindo com filhos eram mais freqüentes no Brasil.

As informações socioeconômicas estão na tabela 2. Os dados referentes à pobreza não são comparáveis nos dois países, por diferenças conceituais. Utilizando o conceito dos Estados Unidos, $11 \%$ da população idosa daquele país foram considerados pobres. Nos cálculos brasileiros, 23\% das pessoas com 60 anos ou mais de idade eram pobres. Entre os seis indicadores socioeconômicos, o que apresentou melhor comparabilidade foi a "taxa de atividade", que evidenciou valores semelhantes para as mesmas idades nos dois países.

Alguns indicadores de saúde constantes da tabela 3 mostraram valores próximos para os dois países. Esse é o caso da esperança de vida aos 65 anos (indicador 12), que foi de 17 anos no Brasil e de 18 anos nos Estados Unidos. No Brasil, as taxas de mortalidade por doença cerebrovascular e por diabetes foram mais elevadas. Os Estados Unidos, por sua vez, apresentaram altas taxas de mortalidade e morbidade por neoplasias malignas e de morbidade auto-referida por artrite. 
TABELA 1. Indicadores demográficos da população com 65 ou mais anos de idade, Brasil e Estados Unidos, 2000ª

\begin{tabular}{|c|c|c|c|}
\hline Brasil & & & Estados Unidos \\
\hline Número de idosos (em milhões) & 10 & 35 & Number of elderly age 65 and older (million) \\
\hline Proporção na população (\%) & 6 & 13 & Percentage of the population (\%) \\
\hline Composição racial e étnica (\%) & & & Racial and ethnic composition (\%) \\
\hline Branca & 62 & 84 & Non-hispanic white \\
\hline$\ldots$ & $\ldots$ & 6 & Hispanic \\
\hline Parda & 30 & $\ldots$ & $\ldots$ \\
\hline Preta & 7 & 8 & Non-Hispanic black \\
\hline Amarela & 1 & 2 & Non-Hispanic Asian and Pacific Islander \\
\hline Indígena & 0,4 & 0,4 & Non-Hispanic American Indian and Alaska native \\
\hline Estado civil (\%) & & & Marital status (\%) \\
\hline Casados & 47 & 57 & Married \\
\hline Separados e divorciados & 3 & 7 & Divorced \\
\hline Viúvos & 33 & 33 & Widowed \\
\hline Solteiros & 16 & 4 & Never married \\
\hline Grau de escolaridade $(\%)^{b, c}$ & & & Educational attainment (\%) \\
\hline Superior & 4 & 15 & Bachelor's degree or higher \\
\hline$\ldots$ & $\ldots$ & 67 & High school diploma or higher \\
\hline Médio & 6 & $\ldots$ & $\ldots$ \\
\hline Básico & 90 & $\ldots$ & $\ldots$ \\
\hline Arranjo familiar (\%) ${ }^{\mathrm{d}}$ & & & Living arrangement (\%) \\
\hline Casal residindo com ou sem filhos & 61 & 57 & With spouse \\
\hline Idoso residindo com filhos ou parentes & 26 & 14 & With other relatives or non-relatives \\
\hline Idoso residindo sozinho & 13 & 29 & Alone \\
\hline
\end{tabular}

Não foi encontrado, no Brasil, registro de comprometimento de memória em idosos que tivesse abrangência nacional. A auto-avaliação da saúde revelou que $84 \%$ dos brasileiros idosos consideravam a sua saúde entre regular e muito boa. Nos Estados Unidos, 72\% consideravam a sua saúde entre boa e muito boa. A taxa de incapacidade de realizar atividades nas últimas 2 semanas por motivo de saúde alcançou $14 \%$ dos idosos brasileiros. Nos Estados Unidos, as incapacidades crônicas atingiam $21 \%$ dos idosos. A depressão também foi mais elevada entre os idosos norte-americanos.

Em relação aos hábitos de vida e aos fatores de risco (tabela 4), a situação foi mais favorável nos Estados Unidos, com exceção da cobertura vacinal contra a influenza: $67 \%$ no Brasil e $50 \%$ naquele país. Não foram encontrados dados brasileiros sobre atividade social e crimes violentos, na forma como foram definidos na publicação norte-americana.
Quanto aos indicadores relativos à assistência à saúde (tabela 5), nota-se que a maioria não encontrou correspondência exata. Verificou-se que, em ambos os países, os que ganhavam mais gastavam proporcionalmente menos com saúde (indicador 25). Os brasileiros gastavam relativamente mais com produtos farmacêuticos (indicador 26). Os idosos norte-americanos tinham maior acesso e utilizavam mais a rede de serviços (indicadores 28 e 29). A utilização de domicílios coletivos e de assistência domiciliar foi mais expressiva nos Estados Unidos.

\section{DISCUSSÃO}

A interpretação das diferenças observadas entre os indicadores deve ser cautelosa. As informações provenientes de inquéritos e recenseamentos, em ambos os países, tendem a ser de boa qualidade; contudo, admite-se que os dados de registros contínuos como mortalidade e internação hospitalar são menos confiáveis no Brasil por questões de cobertura populacional e por problemas no preenchimento dos registros (5). As diferenças conceituais entre indicadores também dificultam a comparação, especialmente naqueles com maior grau de subjetividade na aferição. Outras limitações em potencial são os diferentes significados dos números, que variam de acordo com o método utilizado para cada indicador, e o fato de os dados se referirem ao período de um único ano, o que impede a avaliação de tendências ou a verificação do caráter típico ou atípico da informação. Quanto à padronização dos dados por idade, ela não foi feita, até porque a proporção dos muito velhos é próxima nos dois países: 1,6\% de idosos acima de 80 anos nos Estados Unidos e 1,1\% no Brasil $(6,19)$.

Os dados demográficos (tabela 1) apresentam menor complexidade de análise quando comparados aos demais. $\mathrm{O}$ entendimento, no entanto, re- 
TABELA 2. Indicadores econômicos da população com 65 ou mais anos de idade, Brasil e Estados Unidos, 2000a

\begin{tabular}{|c|c|c|c|}
\hline Brasil & & & Estados Unidos \\
\hline Pobreza $(\%)^{b}$ & 23 & $\ldots$ & Poverty (\%) \\
\hline Distribuicão conforme renda per capita das & $\cdots$ & 11 & $\cdots$ \\
\hline famílias com idosos $(\%)^{c}$ & & & Income distribution (\%) \\
\hline$\ldots$ & $\ldots$ & 2 & Extreme poverty \\
\hline$\ldots$ & $\ldots$ & 8 & Poverty \\
\hline$\ldots$ & $\ldots$ & 27 & Low income \\
\hline$\ldots$ & $\ldots$ & 35 & Medium income \\
\hline$\ldots$ & $\ldots$ & 28 & High income \\
\hline < um quarto de salário mínimo & 2 & $\ldots$ & $\ldots$ \\
\hline De um quarto $\mathrm{a}<$ meio salário mínimo & 7 & $\ldots$ & $\ldots$ \\
\hline De meio a $<1$ salário mínimo & 23 & $\ldots$ & $\ldots$ \\
\hline De 1 a < 2 salários mínimos & 36 & $\ldots$ & $\ldots$ \\
\hline 2 salários mínimos ou mais & 33 & $\ldots$ & $\ldots$ \\
\hline Fontes de renda $(\%)^{d}$ & & & Sources of income $(\%)$ \\
\hline Aposentadoria & 65 & 38 & Social security \\
\hline Trabalho & 20 & 21 & Earnings \\
\hline$\ldots$ & $\ldots$ & 20 & Asset income \\
\hline Pensão & 5 & 19 & Pensions \\
\hline Aluguéis & 9 & $\ldots$ & $\ldots$ \\
\hline Outros & 0,2 & 2 & Other \\
\hline Valor da renda líquida $(\%)^{\mathrm{e}}$ & $\ldots$ & 158 & Net worth (US\$) \\
\hline < 2 salários mínimos & 62 & $\ldots$ & $\ldots$ \\
\hline De 2 a 5 salários mínimos & 22 & $\ldots$ & $\ldots$ \\
\hline Acima de 5 salários mínimos & 16 & $\ldots$ & $\ldots$ \\
\hline Taxa de atividade (\% por idade $)^{\dagger}$ & & & Participation in the labor force ( $\%$ for age) \\
\hline 65 a 69 & 27 & 23 & 65 a 69 \\
\hline 70 anos e mais & 12 & 9 & 70 years and over \\
\hline Despesas com outros imóveis $(\%)^{g}$ & & & Housing expenditures (\%) \\
\hline 0 a 2 salários mínimos & 0,6 & $\ldots$ & $\ldots$ \\
\hline 2 a 5 salários mínimos & 0,6 & $\ldots$ & $\ldots$ \\
\hline 5 e mais salários mínimos & 16 & & $\ldots$ \\
\hline$\ldots$ & $\ldots$ & 36 & Lowest fifth \\
\hline$\ldots$ & $\ldots$ & 35 & Second fifth \\
\hline$\ldots$ & $\ldots$ & 29 & Third fifth \\
\hline$\ldots$ & $\ldots$ & 28 & Fourth fifth \\
\hline$\ldots$ & $\ldots$ & 26 & Highest fifth \\
\hline
\end{tabular}

${ }^{a}$ Com base nos critérios de 6 a 11 de Federal Interagency Forum on Aging-Related Statistics (6). A indicação . . . se refere a casos sem categorias equivalentes nos dois países.

${ }^{b}$ Conforme Barros et al. (24). Dados referentes à população com 60 anos e mais de idade em 2003.

c Instituto Brasileiro de Geografia e Estatística (15). Com base em salário mínimo de $\mathrm{R} \$ 130,00$. O cálculo não inclui as áreas rurais da Região Norte.

d Conforme Almeida (23).

e Conforme Romero (29). Renda líquida em salários mínimos de $\mathrm{R} \$ 130,00$.

f Conforme Camarano et al. (33).

g Conforme Almeida (23). Inclui: aquisição de imóveis, aluguel de imóvel, aluguel de garagem, condomínio, imposto predial, segurança contra roubo e incêndio, energia elétrica, gás, taxas diversas, aquisição de sepultura ou título de clube, imposto territorial rural, aluguel de terreno para sepultura, mudança, valor do imóvel adquirido em primeira locação e valor do imóvel adquirido após já ter sido usado. Proporção da despesa com imóveis nos gastos totais mensais da população com 60 ou mais anos de idade, segundo classe de rendimento em salários mínimos.

quer ressalvas. Deve-se ter em vista, por exemplo, a dificuldade de mensurar etnias ou a observação de que há mais idosos solteiros no Brasil proporcionalmente aos Estados Unidos, que pode decorrer de uma resposta de indivíduos que consideram como casamento apenas a união formal (19).

Quanto aos indicadores socioeconômicos (tabela 2), ressalte-se que esses dados não permitem inferir, por exemplo, que a proporção de idosos pobres é duas vezes maior no Brasil. Essas taxas dizem respeito a diferentes condições, originadas de países com distintas características econômicas. Sendo assim, cada coeficiente tem valor interno no país em que foi criado. Se fosse realizada a padronização dos valores monetários para fins de comparação, os valores econômicos seriam outros. Os benefícios institucionais oferecidos nos países também interferem nesses indicadores, fazendo com que cada local apresente características sui generis $(6,11)$. Para realçar a diferença de conceitos nas comparações, é conveniente relembrar o indicador de pobreza. A pobreza é um importante determinante das condições 
TABELA 3. Indicadores da condição de saúde da população com 65 ou mais anos de idade, Brasil e Estados Unidos, 2000ª

\begin{tabular}{|c|c|c|c|}
\hline \multicolumn{2}{|l|}{ Brasil } & \multicolumn{2}{|r|}{ Estados Unidos } \\
\hline $\begin{array}{l}\text { Esperança de vida aos } 65 \text { anos (em anos) })^{b} \\
\text { Mortalidade (por } 100000 \text { hab }^{c}\end{array}$ & 17 & 18 & $\begin{array}{l}\text { Life expectancy at age of } 65 \text { (in years) } \\
\text { Mortality rate (per } 100000 \text { ) }\end{array}$ \\
\hline Doenças do aparelho circulatório & 1688 & 1832 & Heart disease \\
\hline Doença cerebrovascular & 559 & 426 & Stroke \\
\hline Diabetes & 224 & 141 & Diabetes \\
\hline Neoplasias & 615 & 1133 & Cancer \\
\hline Pneumonia & 168 & 237 & Pneumonia \& influenza \\
\hline Doença pulmonar obstrutiva crônica & 249 & 281 & Chronic obstructive pulmonary disease \\
\hline Morbidade auto-referida (\%) ${ }^{\mathrm{d}, \mathrm{e}}$ & & & Chronic health status (\%) \\
\hline Hipertensão & 44 & 45 & Hypertension \\
\hline Doenças do coração & 19 & 21 & Heart disease \\
\hline Diabetes & 10 & 12 & Diabetes \\
\hline Câncer & 1 & 19 & Cancer \\
\hline Artrite ou reumatismo & 38 & 58 & Arthrits \\
\hline Comprometimento de memória & & 16 & Memory impairment $(\%)^{\dagger}$ \\
\hline Depressão auto-referida (\%) ${ }^{9}$ & 12 & 18 & Depressive symptoms (\%) \\
\hline Percepção da saúde (\%) $)^{\mathrm{d}, \mathrm{e}, \mathrm{h}}$ & 84 & 72 & Self-rated health status (\%) ${ }^{i}$ \\
\hline Incapacidades (\%) $)^{\mathrm{d}, \mathrm{e}, \mathrm{j}}$ & 14 & 21 & Disability (\%) \\
\hline \multicolumn{4}{|c|}{$\begin{array}{l}\text { a Com base nos critérios de } 12 \text { a } 18 \text { de Federal Interagency Forum on Aging-Related Statistics (6). A in } \\
\text { países. } \\
\text { b Instituto Brasileiro de Geografia e Estatística (19). Tábuas completas de mortalidade, } 2000 . \\
\text { c Rede Interagencial de Informações para a Saúde (14). } \\
\text { d Conforme Lima e Costa et al. (26). } \\
\text { e Dado para } 60 \text { anos. } \\
\text { f Inclui moderate e severe (moderado e grave). } \\
\text { g Conforme Camarano et al. (33). } \\
\text { h Inclui regular, bom, muito bom. } \\
\text { i Inclui good e very good (bom e muito bom). } \\
\text { j Pessoas que deixaram de realizar atividades habituais nas últimas } 2 \text { semanas por motivo de saúde. }\end{array}$} \\
\hline
\end{tabular}

TABELA 4. Indicadores dos hábitos de vida e fatores de risco da população com 65 ou mais anos de idade, Brasil e Estados Unidos, $2000^{\mathrm{a}}$

\begin{tabular}{|c|c|c|c|}
\hline Brasil & & & Estados Unidos \\
\hline Atividade social & $\ldots$ & 64 & Social activity (\%) \\
\hline Insuficientemente ativos (\%) ${ }^{\mathrm{b}}$ & 42 & 34 & Sedentary lifestyle (\%) \\
\hline Cobertura vacinal por influenza $(\%)^{c}$ & 67 & 50 & Vaccinated against influenza (\%) ${ }^{d}$ \\
\hline Mamografia nos últimos 2 anos (\%) & 37 & 55 & Mammography in the past 2 years (\%) \\
\hline Qualidade da dieta $(\%)^{b, f}$ & 7 & 21 & Dietary quality $(\%)^{g}$ \\
\hline Crimes violentos & $\ldots$ & 3 & Violent crime (per 1 000) \\
\hline \multicolumn{4}{|c|}{$\begin{array}{l}\text { a Com base nos critérios de } 19 \text { a } 24 \text { de Federal Interagency Forum on Aging-Related Statistics (6). A indicação ... se refere a casos sem categorias equivalentes } \\
\text { nos dois países. } \\
\text { b Brasil, Ministério da Saúde (13). } \\
\text { c Ministério da Saúde, Programa Nacional de Imunizações (41). } \\
\text { d Dados relativos ao ano de } 1995 . \\
\text { e Instituto Brasileiro de Geografia e Estatística (17). } \\
\text { f Indivíduos que consomem frutas, legumes ou verduras cinco ou mais vezes por dia. } \\
\text { g Dado para pessoas com } 45 \text { e mais anos de idade. Inclui good e very good (bom e muito bom). }\end{array}$} \\
\hline
\end{tabular}

de vida em todo o planeta, pois aumenta o risco de a pessoa não ter suprimento adequado de alimento, moradia, assistência à saúde e outras necessidades básicas $(6,24)$. A medida oficial de pobreza nos Estados Unidos se baseia na comparação da renda familiar com um conjunto de padrões, que variam de acordo com a composição e o tamanho da família. Dessa maneira, há $11 \%$ de pobres entre os que estão com 65 anos ou mais de idade $(6,30)$. Considerando-se esse percentual, inferiu-se um número absoluto de 4 milhões de idosos pobres nos Estados Unidos.

No Brasil, foram considerados pobres todos os que tinham renda domi- 
TABELA 5. Indicadores de assistência à saúde para a população com 65 ou mais anos de idade, Brasil e Estados Unidos, 2000a

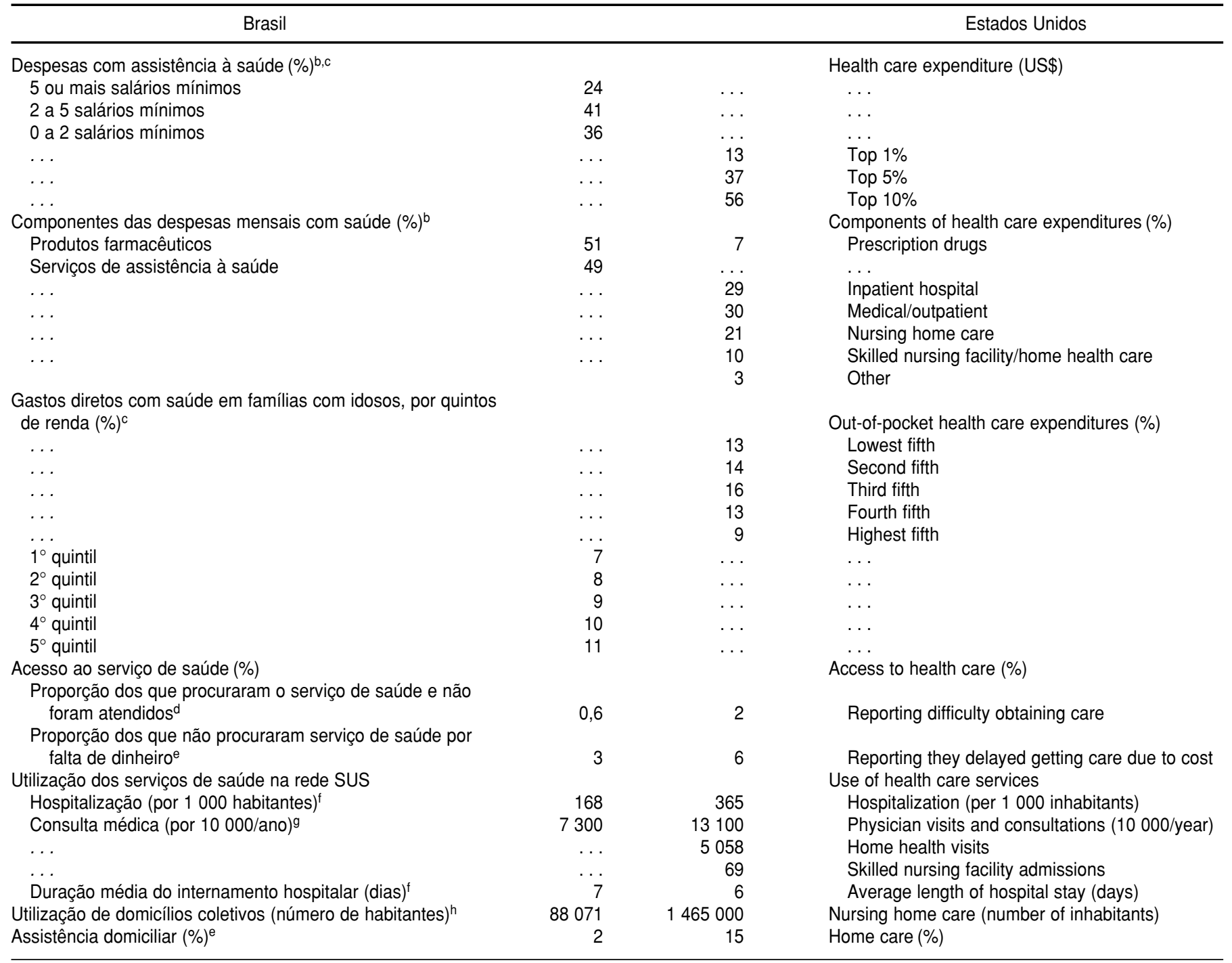

${ }^{a}$ Com base nos critérios de 25 a 31 de Federal Interagency Forum on Aging-Related Statistics (6). A indicação ... se refere a casos sem categorias equivalentes nos dois países.

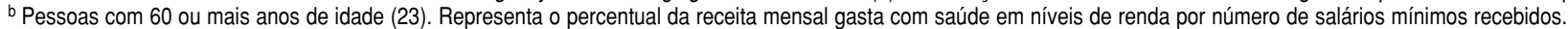

${ }^{c}$ Instituto Brasileiro de Geografia e Estatística (16).

d Conforme Lima e Costa et al. (26).

e Instituto Brasileiro de Geografia e Estatística (15).

† Rede Interagencial de Informações para a Saúde (14).

${ }^{9}$ Conforme Pinheiro et al. (28). Setenta e três por cento das pessoas realizaram pelo menos uma consulta médica no ano

${ }^{\mathrm{h}}$ Conforme Camarano et al. (1).

ciliar per capita inferior a uma dada linha de pobreza relacionada aos percentuais do salário mínimo do País. A linha de pobreza estabelecida tem variações regionais, pois as condições econômicas são distintas em diferentes regiões dentro do Brasil. A estrutura econômica dos idosos também varia em função da maior probabilidade de surgimento de gastos elevados e inesperados, principalmente em decorrência de problemas com a saúde (24). Com base nesses dados, os pesquisadores encontraram $30 \%$ de pobres na população brasileira, sendo que $23 \%$ dos que tinham 60 anos ou mais se en- contravam nessa condição, o que equivalia a 3 milhões de idosos pobres no Brasil (para uma população com 60 anos ou mais de idade estimada em 14,5 milhões em 2000) $(19,24)$.

Verificou-se uma diferença pequena na esperança de vida entre os Estados Unidos e o Brasil. É possível que esse 
indicador tenha baixo poder discriminador, pois países com diferentes estágios de desenvolvimento podem apresentar taxas semelhantes, como já foi mostrado em comparações entre a Suécia e a Índia (31) ou entre a Bélgica, a Finlândia e o Brasil (32).

Os dados de mortalidade no Brasil podem não ser tão precisos quanto os dos Estados Unidos. Estima-se que o Sistema de Informação sobre Mortalidade (SIM) do Ministério da Saúde tenha cobertura média no País de $80 \%$, mas de apenas 60\% nas regiões Norte e Nordeste (5). Essa situação pode resultar em subnotificação dos diagnósticos das causas de mortalidade, o que elevaria ainda mais as taxas brasileiras de mortalidade por doença cerebrovascular e por diabetes expressas na tabela 3. Sobre a qualidade da informação, deve-se também considerar a elevada taxa de causas mal definidas em idades mais avançadas no Brasil, o que torna difícil a interpretação das causas de morte nessa faixa etária (33). As causas mal definidas podem estar relacionadas a erros de diagnóstico e a falta de conhecimento sobre as doenças em idosos (34), o que pode interferir nos registros de doenças alterando a quantidade dos resultados apresentados na tabela 3 .

Os países estudados apresentaram freqüências semelhantes de enfermidades como hipertensão, diabetes e doenças do coração. Os dados de morbidade de ambos os países (tabela 3) são autoreferidos. Trabalhos recentes no Brasil têm mostrado boa sensibilidade e, sobretudo, ainda maior especificidade para a detecção de doenças crônicas auto-referidas em inquéritos (35). Os dados de um estudo de base populacional brasileira mostram que a prevalência da hipertensão auto-referida na população $(27 \%)$ foi só pouco maior do que a determinada por meio de três medidas da pressão arterial e história de tratamento para a mesma (23\%) (35).

Os idosos dos Estados Unidos têm maior acesso e utilizam mais a rede de serviços. Sendo assim, conhecem mais os seus agravos à saúde e os revelam nas entrevistas. Além disso, têm maior escolaridade, o que influencia não só na busca por serviços como no próprio re- lato sobre sua saúde (36). Isso pode explicar, em parte, a grande diferença na freqüência auto-referida de câncer: 1\% no Brasil e 19\% nos Estados Unidos.

Não há registros com alcance nacional sobre o comprometimento da memória dos idosos brasileiros (37). A literatura mostra que, na população idosa, esse sintoma pode fazer parte do quadro clínico da síndrome demencial, acarretando diminuição da qualidade de vida e maior demanda por serviços de saúde (37). Isso reforça a necessidade de incluir esse tema nos inquéritos realizados no Brasil.

Observa-se que, nos dois países, mais da metade das pessoas idosas sente-se em condição de saúde entre regular e muito boa. A auto-avaliação da saúde reflete a percepção integrada do indivíduo sobre si mesmo e inclui as dimensões biológica, psicológica e social. O uso da auto-avaliação da saúde em inquéritos populacionais vem crescendo, depois de ter sido evidenciado que a mesma pode predizer mortalidade (38).

As informações sobre incapacidades também são utilizadas no monitoramento da saúde e do bem-estar da população idosa (7). Ocorrem incapacidades em cerca de $10 \%$ das pessoas de todas as idades e até cinco vezes mais nas amostras só de idosos, sendo essa elevação mais acentuada naqueles indivíduos com 80 anos ou mais de idade (7). A incapacidade de realizar atividades nas últimas 2 semanas por motivo de saúde foi referida por $14 \%$ dos idosos brasileiros. Nos Estados Unidos, $21 \%$ das pessoas com 65 anos ou mais de idade relataram ser portadoras de incapacidades crônicas. É possível que a baixa freqüência detectada no Brasil se deva a questões de aferição (7).

Nos dois países, a maior parte dos idosos relata a presença de doença e também de bem-estar. Só uma minoria se declara incapaz. Esse achado confirma que as pessoas idosas podem se sentir bem mesmo tendo doenças (39).

Os idosos norte-americanos tinham maiores taxas de atividade física e de qualidade da dieta, o que pode estar associado a elementos culturais e socioeconômicos, além da percepção dos benefícios advindos da adoção de prá- ticas preventivas (40). A mais elevada taxa de cobertura vacinal por influenza, no Brasil, pode ser produto das campanhas periódicas no País $(41,42)$. Embora não se tenha o equivalente brasileiro, os norte-americanos apresentaram altas taxas de atividade social $(64 \%)$. Esse indicador inclui visitas a amigos e parentes, atividades em igrejas ou semelhantes e trabalho voluntário em associações diversas, o que está relacionado à cultura.

Os indicadores de crimes violentos, não encontrados com a mesma dimensão no Brasil, são estudados nos Estados Unidos por meio de pesquisas nacionais (National Crime Victimization Survey e Uniform Crime Reports) que incluem o registro de estupro, agressão sexual, assalto, agressão simples e agregada, roubo pessoal, roubo de propriedade, arrombamento de casa e roubo de veículo motorizado. As vítimas são solicitadas a fornecer as características dos crimes e até mesmo informar se relataram o incidente para a polícia (6).

Para alguns indicadores de assistência à saúde, não existe equivalência exata nas categorias de análise. Trata-se de uma área que demanda melhor padronização para permitir comparações.

Os indicadores de assistência à saúde são influenciados pelos aspectos econômicos da população estudada. Os idosos que têm maior renda também têm mais acesso aos serviços de saúde e mais facilidade de usá-los (36). Concomitantemente, os idosos de baixa renda têm acesso limitado à alimentação saudável, saneamento básico e informações sobre promoção da saúde, prevenção das doenças e seu tratamento. Sendo assim, a carga de doença resultante reflete também a condição econômica e influencia a procura pelos serviços de saúde (43). Outro aspecto econômico a ser destacado é o peso dos gastos com saúde no orçamento doméstico. À medida que aumenta a renda familiar, diminui o peso dos gastos com saúde, ou seja, os mais pobres gastam proporcionalmente mais com saúde em relação ao total da sua receita mensal (43).

A informação sobre a utilização hospitalar está baseada em dados institucionais, de modo que a cobertura se 
restringe aos que têm acesso a esse tipo de serviço. Em todos os sistemas brasileiros, há sub-registros que variam no espaço e no tempo. A tendência é a melhoria da cobertura e da qualidade com o passar do tempo. No Brasil, foram consideradas apenas as internações em unidades hospitalares vinculadas ao Sistema Único de Saúde (SUS), representando cerca de $60 \%$ do total. Há também o problema da contagem cumulativa de internações de um mesmo paciente (5). Nos Estados Unidos, os dados referem-se ao Medicare, um programa de seguro-saúde que cobre quase a totalidade de pessoas de 65 anos de idade ou mais (44). Sendo assim, a análise dos dados oriundos dessas duas instituições também deve ser avaliada com cautela.

A evolução longitudinal dos 31 indicadores, nos Estados Unidos, revela avanços e disparidades (45). Os idosos estão vivendo mais com menos incapacidades e em melhores condições econômicas. Entretanto, esses avanços não têm atingido homogeneamente a todos os que envelhecem. Os descendentes de africanos e de hispânicos continuam com piores condições de vida. A expectativa de vida cresce continuadamente, as taxas de mortalidade decrescem, principalmente aquelas relacionadas às causas de morte por doenças cardiovasculares. As enfermidades crônicas estão menos relaciona- das com incapacidades graves. $\mathrm{O}$ comprometimento da memória é crescente com o aumento de idade, principalmente depois dos 80 anos; isso também acontece com os sintomas depressivos, mesmo sem um aumento franco nos mais longevos. Há um decréscimo de incapacidades nos idosos dos Estados Unidos na última década; as mulheres são as mais atingidas, assim como os afro-descendentes. Os dados sobre fatores de risco e hábitos de vida revelam tendências positivas. As maiores taxas de vacinações, mamografias e dietas favoráveis comprovam essa afirmação. A análise das informações relacionadas ao acesso e à utilização de serviços de saúde revela mudanças nas condições de saúde dos norte-americanos que envelhecem, confirmando a tendência dos demais indicadores e mantendo a ausência de eqüidade e a presença de disparidades entre os menos favorecidos economicamente (45).

A avaliação histórica de informações sobre os brasileiros que envelhecem no Brasil sinaliza trajetória também positiva (1). Estudos permitem o acompanhamento da autopercepção da saúde, das doenças crônicas auto-referidas, da capacidade funcional e do acesso e utilização dos serviços de saúde. Os dados mostram que, entre 1998 e 2003, anos de realização de inquéritos no Brasil, houve uma redução no relato dos idosos quanto a doenças, algumas crônicas, e aumento do número de consultas médicas. Paralelamente, houve menos registros de internações hospitalares. A freqüência dos idosos que referiram incapacidades diminuiu e a dos que declararam sua própria saúde como boa e muito boa aumentou (1).

Entre os desafios a serem enfrentados, encontram-se a necessidade de melhor sistematização das informações, a sua ampla divulgação e o seu uso para o planejamento, a gestão e a avaliação de políticas voltadas para a saúde do idoso. Cabe destacar que, no Brasil, mesmo com muito ainda a ser feito, já existem numerosas informações que permitem acompanhar a evolução da saúde do idoso. É provável que a situação seja semelhante em muitos países em vias de desenvolvimento.

Em conclusão, a partir de um grupo de indicadores utilizados para avaliar o bem-estar dos idosos norte-americanos, o presente estudo revelou, no Brasil, a existência de dados para a maioria (90\%) dos indicadores da lista norteamericana, sem investimento especial em pesquisa para a produção de novos dados. Foram realizadas apenas consultas em bases eletrônicas e, por vezes, a reanálise das informações. Os dados encontrados sugerem que, se o poder discriminatório dos indicadores utilizados for de fato adequado, as condições de saúde dos idosos estão próximas nos dois países pesquisados.

\section{REFERÊNCIAS}

1. Camarano AA, Kanso S, Pasinato MT, Mello JLE. Idosos brasileiros: indicadores de condição de vida e acompanhamento de políticas. Brasília: Presidência da República, Subsecretaria de Direitos Humanos; 2005.

2. Pereira MG, Rebouças M. Indicadores de saúde para idosos. Em: Freitas EV, Py L, Cançado FAX, Doll J, Gorzoni ML. Tratado de geriatria e gerontologia. $2^{\mathrm{a}}$ ed. Rio de Janeiro: Guanabara Koogan; 2006. Pp. 131-8.

3. Special senate committee on aging first interim report, 2007. The Federal Role - Interim Report. Ontário: Parliament of Canada; 2007. Disponível em: http://www.parl.gc.ca/39/ 1/parlbus/commbus/senate/com-e/agei-e/ rep-e/repintfeb07-e.htm\#Order_of_Reference. Acessado em janeiro de 2008.
4. Rede Interagencial de Informações para a Saúde (RIPSA), Comitê Temático Interdisciplinar (CTI). Saúde do idoso. Relatório final. Brasília: Ministério da Saúde; 2006. Disponível em: http://www.opas.org.br/ripsa/ UploadArq/relato_final_com_fichas[1].pdf. Acessado em novembro de 2006.

5. Rede Interagencial de Informações para a Saúde (RIPSA). Indicadores básicos para a saúde no Brasil: conceitos e aplicações. Brasília: Organização Pan-Americana da Saúde; 2002. Disponível em: http://www.opas.org. br/sistema/arquivos/matriz.pdf. Acessado em janeiro de 2006.

6. Federal Interagency Forum on Aging-related Statistics 2005. Older Americans 2000: key indicators of well-being. Federal Interagency
Forum on Aging-Related Statistics. Washington, DC: US Government Printing Office; 2000. Disponível em: http://www.agingstats. gov. Acessado em fevereiro de 2006.

7. Barbotte E, Guillemin F, Chau N; Lorhandicap Group. Prevalence of impairments, disabilities, handicaps and quality of life in the general population: a review of recent literature. Bull World Health Organ. 2001;79(11): 1047-55.

8. Envejecimiento y salud: un cambio de paradigma. Rev Panam Salud Publica. 2000;7(1): 60-7.

9. Banoob SM. Training needs and services for elderly care in developing countries: models from Romania, Barbados and Kuwait. Int J Aging Hum Dev. 1992;34(2):125-34. 
10. World Health Organization. The uses of epidemiology in the study of the elderly: report of a WHO scientific group on the epidemiology of aging. Genebra: WHO; 1984. (Technical Report Series no. 706)

11. Brasil, Ministério da Saúde. Estatuto do idoso. Brasília: Ministério da Saúde; 2003.

12. Rede Interagencial de Informações para a Saúde (RIPSA). Indicadores e Dados Básicos (IDB), Brasil, 2005. Brasília: Ministério da Saúde; 2005. Disponível em: http://tabnet. datasus.gov.br/cgi/idb2005/folder.htm. Acessado em janeiro de 2007.

13. Brasil, Ministério da Saúde, Secretaria de Vigilância em Saúde, Secretaria de Atenção à Saúde, Instituto Nacional de Câncer, Coordenação de Prevenção e Vigilância. Inquérito domiciliar sobre comportamentos de risco e morbidade referida de doenças e agravos não transmissíveis: Brasil, 15 capitais e Distrito Federal, 2002-2003. Rio de Janeiro: Ministério da Saúde; 2004. Disponível em: http://www. inca.gov.br/inquerito/docs/completa.pdf. Acessado em fevereiro de 2006

14. Rede Interagencial de Informações para a Saúde (RIPSA). Indicadores e Dados Básicos Brasil. Brasília: Ministério da Saúde; 2006. Disponível em: http://tabnet.datasus.gov. br/cgi/idb2006/matriz.htm. Acessado em janeiro de 2006

15. Instituto Brasileiro de Geografia e Estatística (IBGE), Departamento de Emprego e Rendimento. Acesso e utilização de serviços de saúde: 1998: Brasil/IBGE. Rio de Janeiro: IBGE; 2000. Disponível em: http://www. ibge.gov.br/home/estatistica/populacao/ trabalhoerendimento/pnad98/saude/saude. pdf. Acessado em janeiro de 2006.

16. Instituto Brasileiro de Geografia e Estatística (IBGE), Coordenação de Índices de Preços. Pesquisa de orçamentos familiares 2002-2003: primeiros resultados: Brasil e grandes regiões/ IBGE. Rio de Janeiro: IBGE; 2004. Disponível em: http://www.ibge.gov.br/home/ estatistica/populacao/condicaodevida/pof/2 002/pof2002.pdf. Acessado em janeiro de 2006.

17. Instituto Brasileiro de Geografia e Estatística (IBGE), Coordenação de Trabalho e Rendimento. Acesso e utilização de serviços de saúde: 2003. Rio de Janeiro, IBGE; 2005. Disponível em:http://www.ibge.gov.br/home/ estatistica/populacao/trabalhoerendimento/ pnad2003/saude/default.shtm. Acessado em janeiro de 2006.

18. Rebouças M. Indicadores de saúde dos idosos: comparação entre o Brasil e os Estados Unidos [dissertação de mestrado]. Brasília: Universidade Católica de Brasília; 2006. Pp. 73-80. Disponível em: http://www.bdtd. ucb.br/tede/tde_busca/arquivo.php?cod Arquivo $=484$. Acessado em março de 2008.

19. Instituto Brasileiro de Geografia e Estatística. Rio de Janeiro: IBGE; 2001. Disponível em: http://www.ibge.gov.br/home/estatistica/ populacao/censo2000/default.shtm. Acessado em março de 2006.

20. Rebouças M. Indicadores de saúde dos idosos: comparação entre o Brasil e os Estados Unidos [dissertação de mestrado]. Brasília: Universidade Católica de Brasília; 2006. Pp.
90. Disponível em: http:/ /www.bdtd.ucb.br/ tede/tde_busca/arquivo.php?codArquivo= 484 . Acessado em março de 2008.

21. Rebouças M. Indicadores de saúde dos idosos: comparação entre o Brasil e os Estados Unidos [dissertação de mestrado]. Brasília: Universidade Católica de Brasília; 2006. Pp. 128. Disponível em: http://www.bdtd.ucb. $\mathrm{br} /$ tede/tde_busca/arquivo.php?cod Arquivo=484. Acessado em março de 2008.

22. Rebouças M. Indicadores de saúde dos idosos: comparação entre o Brasil e os Estados Unidos [dissertação de mestrado]. Brasília: Universidade Católica de Brasília; 2006. Pp. 136. Disponível em: http://www.bdtd.ucb. br/tede/tde_busca/arquivo.php?cod Arquivo=484. Acessado em março de 2008.

23. Almeida AN. Determinantes do consumo de famílias com idosos e sem idosos com base na Pesquisa de Orçamentos Familiares 1995/96 [dissertação]. São Paulo: Universidade de São Paulo; 2002.

24. Barros RP, Mendonça R, Santos D. Incidência e natureza da pobreza entre idosos no Brasil. Rio de Janeiro: Instituto de Pesquisa Econômica Aplicada; 1999. (Texto para discussão $\left.n^{\circ} 686.\right)$

25. Leite IC, Schramm JMA, Gadelha AMJ, Valente JG, Campos MR, Portela MC, et al. Comparação das informações sobre a prevalência de doenças crônicas obtidas pelo suplemento saúde da PNAD/98 e as estimadas pelo estudo Carga de Doenças no Brasil. Cienc Saude Coletiva. 2002;7(4):733-41.

26. Lima Costa MF, Barreto S, Giatti L. A situação socioeconômica afeta igualmente a saúde de idosos e adultos mais jovens no Brasil? Um estudo utilizando dados Pesquisa Nacional por Amostra de Domicílio - PNAD/98. Cienc Saude Coletiva. 2002;7(4):813-24

27. Lima Costa MF, Barreto SM, Giatti L. Condição de saúde, capacidade funcional, uso de serviços de saúde e gastos com medicamentos da população idosa brasileira: um estudo descritivo baseado na Pesquisa Nacional por Amostra de Domicílio. Cad Saude Publica. 2003;19(3):735-43.

28. Pinheiro RJ, Viacava F, Travasso C, Brito AS Gênero, morbidade acesso e utilização de serviços de saúde no Brasil. Cienc Saude Coletiva. 2002;7(4):687-707.

29. Romero DE. Diferenças de gênero no impacto do arranjo familiar no status de saúde dos idosos brasileiros. Cienc Saude Coletiva. 2002; 7(4):777-94.

30. US Census Bureau. Census 2000. Washington DC; 2000. Disponível em: www.census.gov/ main/www/cen2000.html. Acessado em novembro de 2006

31. Camarano AA, Medice AC, Oliveira F, Beltrão KI. Aposentadoria e esperança de vida. Conj Econ. 1991;45:41-4.

32. Morvan MM, Carvalho JAM. Envelhecimento da população e aposentadoria por idade. Anais do VIII Encontro de Estudos Populacionais. Brasília: Associação Brasileira de Estudos Populacionais; 1992. Vol. 2. Pp. 297-308. Disponível em: http://www.abep.nepo. unicamp.br/docs/anais/pdf/1992/t92v02a14. pdf Acessado em maio de 2002.
33. Vasconcelos AMN. A qualidade das estatísticas de óbitos no Brasil. Rev Bras Estudos Pop. 1998;15(1):115-24.

34. Lima Costa MF, Guerra HL, Barreto SM, Guimarães RM. Diagnóstico da situação de saúde da população idosa brasileira: um estudo da mortalidade e das internações hospitalares públicas. Informe Epidemiológico SUS. 2000; 9(1):23-41.

35. Lima Costa MF, Peixoto SV, Firmo JOA. Validade da hipertensão arterial auto-referida e seus determinantes (Projeto Bambuí). Rev Saude Publica. 2004;38(5):637-42.

36. Barreto SM, Kalache A, Giatti L. Does health status explain gender dissimilarity in health use among older adult? Cad Saude Publica. 2006;22(2):347-55.

37. Scazufca M, Cerqueira ATAR, Menezes PR, Prince M, Vallada HP, Myazaki MCOS, et al. Investigações sobre demência nos países em desenvolvimento. Rev Saude Publica. 2002; 36(6):773-8.

38. Idler EL, Benyamini Y. Self-rated health and mortality: a review of twenty-seven community studies. J Health Soc Behav. 1997;38(1): 21-37.

39. Ramos LR. Fatores determinantes do envelhecimento saudável em idosos residentes em centro urbano: Projeto Epidoso. Cad Saude Publica. 2003:19(3):793-8.

40. World Health Organization. The world health report 2002: reducing risks, promoting healthy life. Genebra: WHO; 2002.

41. Ministério da Saúde. Programa Nacional de Imunizações. Disponível em: http://tabnet. datasus.gov.br/cgi/tabcgi.exe?pni/cnv/ cpniuf.def . Acessado em outubro de 2006.

42. Silvestre JA. O impacto da vacinação antiinfluenza na população idosa. Em: Freitas EV, PY L, Néri A, Cançado FAX, Gorzoni ML, Rocha SM. Tratado de geriatria e gerontologia. Rio de Janeiro: Guanabara Koogan; 2002. Pp. 569-73.

43. Silveira FG, Osório RG, Piola S F. Os gastos das famílias com saúde. Cienc Saude Coletiva. 2002;7(4):719-31.

44. Estados Unidos, Department of Health and Human Services. Medicare Program - general information. Overview 2006. Baltimore: Department of Health \& Human Services; 2006. Disponível em: http://www.cms.hhs. gov/MedicareGenInfo/. Acessado em novembro de 2006.

45. Guttman C. Older Americans 2000. New data system that tracks health and well-being finds successes and disparities. Geriatrics. 2000; 55(10):63-6, 69 .

Manuscrito recebido em 28 de março de 2007. Aceito em versão revisada em 3 de setembro de 2007. 


\section{ABSTRACT \\ Health indicators in the elderly: a comparison of Brazil and the United States}

Objective. To identify indicators used in evaluating the population 65 years of age and older in Brazil that are comparable to those used in the United States, and to compare the situation of this age group in the two countries.

Method. This was a descriptive, cross-sectional study, based on secondary data. Brazilian equivalents to the well-being indicators employed in the United States were searched in websites published by government health or planning institutions. Data from national surveys were also employed, and when necessary, data from existing databases were reanalyzed.

Results. Of the 31 indicators used in the United States, an equivalent in Brazil was not found for only three: memory impairment, social activity, and violent crime. In the United States, the number of older individuals was greater; level of education was also higher in this age group. The two countries were similar in terms of health status and life expectancy at 65 years of age. Death rates due to cerebrovascular disease and diabetes were higher in Brazil, while death rates due to malignant neoplasms were higher in the United States. Disabilities and self-reported morbidity due to arthritis and depression were more frequent in the United States. The self-perception of health status as good was higher in Brazil, whereas the indicators related to life habits and risk factors indicated a more favorable situation in the United States.

Conclusions. If the discriminating power of the indicators is truly adequate, the health conditions of the elderly in these two countries are similar.

Key words Indicators, aged, health of the elderly, Brazil, United States of America.

\section{Mantenerse en forma para la vida. Necesidades nutricionales de los adultos mayores}

La Organización Panamericana de la Salud (OPS) reconoce que proteger la salud de los adultos mayores es uno de los principales desafíos que enfrenta la salud pública en el siglo XXI. Una buena alimentación y el mantenerse en forma son maneras de garantizar la salud y el bienestar en la vejez. En este marco, la OPS se complace en presentar su más reciente publicación, Mantenerse en forma para la vida. Necesidades nutricionales de los adultos mayores, obra que representa una fuente autorizada de información sobre la alimentación y actividad física recomendadas para los adultos mayores. En el cuerpo principal de la obra se discuten los aspectos epidemiológicos y sociales del envejecimiento, los cambios funcionales y de salud que se producen al envecejer, los efectos de la actividad física, la evaluación del estado nutricional de los adultos mayores y las pautas nutricionales que deben observarse para envejecer sanamente.

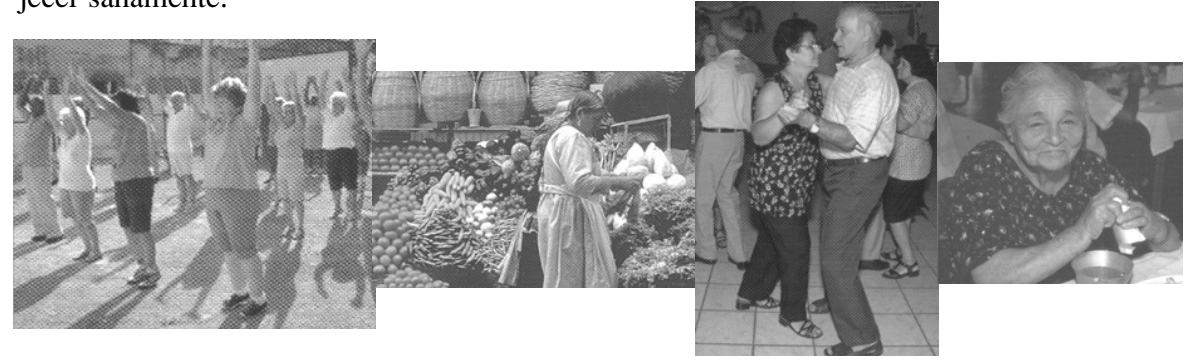

2004, 132 pp., ISBN 9275315957 Código: PC 595 Precios: US\$ 18.00 en América Latina y el Caribe/ US\$25.00 en el resto del mundo

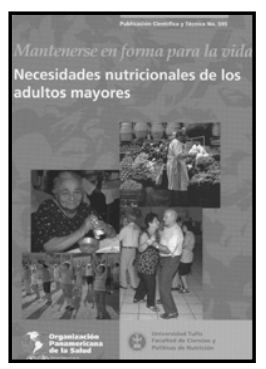

Esta publicación será de mucha utilidad para nutricionistas, médicos generales, gerontólogos, personal de enfermería, proveedores de atención, formuladores de políticas públicas, salubristas y trabajadores sociales. Las recomendaciones específicas sobre la ingesta de nutrientes, el ejercicio y la actividad física también deben interesar a los lectores en general. 